\title{
CIÊNCIA'NATURA
}

\section{Estudo do ciclo diário da Camada Limite Planetária durante a estação chuvosa da Amazônia (GOAmazon 2014/15)}

\author{
${ }^{1}$ Rayonil Gomes Carneiro, ${ }^{1}$ Alice Henkes, ${ }^{2}$ Gilberto Fisch e ${ }^{3}$ Camilla Kassar Borges
}

${ }^{1}$ Instituto Nacional de Pesquisas Espaciais , Brasil

${ }^{2}$ Instituto de Aeronáutica e Espaço, Brasil

${ }^{3}$ Unidade Acadêmica de Ciências Atmosféricas - Universidade Federal de Campina Grande, Brasil

\begin{abstract}
Resumo
Este estudo teve como objetivo caracterizar o padrão do ciclo diário da camada limite planetária na estação chuvosa da Amazônia durante o período de intensa observação realizado na Campanha do Projeto GOAmazon2014/15 (Green Ocean Amazon). A análise inclui dados de radiossondagem e de sensoriamento remoto. De forma geral, os resultados do ciclo diário na estação chuvosa indicam uma camada limite noturna com oscilação na sua profundidade e com uma erosão lenta,da ordem de3 hs. A camada limite convectiva não apresentou um grande desenvolvimento vertical, respondendo aos baixos valores de calor sensivel que são típicos da estação chuvosa. Uma comparação entre as várias técnicas de obtenção da altura da camada limite planetária (observações in situ e de sensoriamento remoto) também é apresentada.
\end{abstract}

Palavras-chave: Erosão da CLN. Fluxos turbulentos. Sensoriamento remoto

\section{Abstract}

In the present study, the evolution the diurnal cycle of planetary boundary layer in the wet season at Amazon region during a period of intense observations carried out in the GOAmazon Project 2014/2015 (Green Ocean Amazon).The analysis includes radiosonde and remote sensing data. In general case, the results of the daily cycle in the wet season indicate a Nocturnal boundary layer with a small oscillation in its depth and with a tardy erosion. The convective boundary layer did not present great depth, responding to the low values of sensible heat of the wet season. A comparison between the different techniques(in situ observations and remote sensing) for estimating the planetary boundary layer is also presented.

Keywords: Nocturnal erosion. Turbulent fluxes. Remote sensing 3 


\section{Introdução}

De acordo com Stull (1988), a Camada Limite Planetária (CLP) é a parte da atmosfera que é diretamente influenciada pela superfície da terra, sendo que os fluxos superficiais de calor sensível e latente desempenham um papel importante no crescimento diurno da CLP. A dinâmica da CLP desempenha um papel fundamental no transporte vertical e na mistura de aerossóis e espécies químicas da superfície para atmosfera livre (OUWERSLOOT et al., 2012). Em função da importância da Amazônia alguns estudos vêm sendo realizados a fim de se obter um melhor conhecimento da CLP na região ao longo dos anos, como em Martin et al. (1988) e Neves e Fisch (2015).

O presente trabalho teve como objetivo investigar as características da CLP na estação chuvosa da Amazônia ao longo do seu ciclo diário e a resposta desta aos fluxos de superfície, usando observações de sensores remotos (Ceilometer, Doppler Lidar, Sodar e radar Wind Profiler) e também observações in situ de radiossondagem. Também é apresentada uma comparação das várias técnicas de obtenção da altura da CLP utilizadas.

\section{Material e Métodos}

O estudo foi realizado no Município de Manacapuru ( $03^{\circ} 18^{\prime}$ 15”'S; 6037'03”O; alt. $60 \mathrm{~m}$ ) no Estado do Amazonas, localizado na região central da Amazônia, cerca de $84 \mathrm{~km}$ distante da capital Manaus. No local esteve instalada a estação denominada T3 (03 $\left.{ }^{\circ} 12^{\prime} 36^{\prime \prime} \mathrm{S} ; 60^{\circ} 36^{\prime} 00^{\prime} \mathrm{O}\right)$, que fez parte campanha do Projeto Observations and Modeling of the Green Ocean Amazon (GoAmazon), mais detalhes em Martin et al., 2016. A estação T3 conteve uma grande quantidade de instrumentos terrestres e de sensoriamento remoto baseados no solo para medições atmosféricas, incluindo medições dos fluxos turbulentos através de um sistema de Eddy Correlation Flux composto de um acoplamento de um anemômetro sônico 3D, modelo WindMaster Pro (Gill Instruments, UK) e um sistema IRGA de CO2/H2O modelo LI-7500 (Li-cor inc., USA). Durante a estação chuvosa, foi realizado um período intensivo de observações (denominada IOP1), que se estendeu do dia 1 de fevereiro a 31 de março de 2014, durante o ápice da estação chuvosa.

\subsection{Radiossonda (RS)}

Durante a campanha do GOAmazon, na IOP1, as RS de alta resolução foram lançadas em cinco horários, as $02,08,11,14$ e 20 Hora Local (HL). A RS forneceu medidas de pressão atmosférica, altitude, temperaturas (do ar e do ponto de orvalho), umidade relativa e determinação da velocidade e direção do vento, com resolução vertical das mediadas de $10 \mathrm{~m}$. Foi calculada, através dos dados obtidos pela RS, a temperatura potencial e, a umidade especifica, os quais foram utilizados para estimar a altura da CLP, através do método da parcela (mais detalhes em Santos et al. (2007).

\subsection{Ceilometer}

As medições da altura da CLP também foram realizadas através de um Ceilometer. Este instrumento fornece medidas do retroespalhamento óptico, com resolução temporal de 16 s (SHUKLA et al., 2014; CARNEIRO; FISCH; KAUFMANN, 2016). Neste trabalho foram realizadas médias de 30 minutos da altura da CLP, a partir das estimativas da altura da CLP realizadas pelas medidas de retroespalhamento.

\subsection{Doppler Lidar}

Também foi utilizado para estimativas da altura da CLP um Doppler Lidar, é um instrumento autônomo de sensoriamento remoto que fornece medições de velocidade radial e de retroespalhamento atenuado no tempo. O Lidar possui capacidade de varredura hemisférica superior, permitindo o mapeamento tridimensional de fluxos turbulentos dentro da CLP. Através da variância da velocidade vertical $\left(\sigma_{w}^{2} \sigma_{w}^{2} \sigma_{w}^{2}\right)$, fornecida pelo Lidar, foi utilizada para determinar a profundidade da CLP, seguindo metodologia de Huang et al. (2017), onde os autores definem a CLP como a camada em que $\sigma_{w}^{2} \sigma_{w}^{2}$ excede um determinado limite $\left(0,1 \mathrm{~m}^{2} \mathrm{~s}^{-2}\right)$

\subsection{Sodar}

No sítio experimental também foi instalado um Mini SODAR (Sound Detection and Ranging). Este equipamento monoestático consiste em uma antena emissora/receptora. A partir do SODAR foram obtidos dados dos perfis vertical da velocidade e direção do vento em intervalos de tempo de $30 \mathrm{~min}$ e com um alcance máximo de $400 \mathrm{~m}$ de altura. Por meio das medições por sensoriamento remoto do SODAR foi calculada a altura da CLP, em sua fase noturna (CLN), através da determinação da altura do vento máximo, conforme sugerido por Neves e Fisch (2011). 


\subsection{Radar Wind Profiler (RWP)}

Finalizando, também foi utilizadas medições do Radar Wind Profiler and RASS (Radio Acoustic Sound System) para medições diretas e contínuas da CLP. Os $R W P$ são radares Doppler para detecção do perfil vertical do vento. Eles trabalham com frequência de $50 \mathrm{MHz}$ a $16 \mathrm{GHz}$. Seguindo metodologia utilizada por Wang et al. (2016), na qual os autores estimaram a altura da CLP através do perfil vertical do índice de refração eletromagnético do RWP, onde o máximo deste índice ocorre na parte superior da CLP.

\section{Resultados e discussão}

Durante o IOP1 da estação chuvosa foi registrado total pluviométrico acumulado de $720 \mathrm{~mm}$ (Figura 1). Entre os horários das 00 e $06 \mathrm{HL}$, a precipitação acumulada foi de $157 \mathrm{~mm}$, o que representou, aproximadamente, $21 \%$ da chuva deste período. Durante o período diurno (entre 06 a $17 \mathrm{HL}$ ) ocorreram as maiores frequências de chuva, acarretando em total acumulado de $450 \mathrm{~mm}$, sendo que mais de $60 \%$ da chuva esteve concentrada neste intervalo. O ano de 2014 apresentou uma precipitação típica da região.

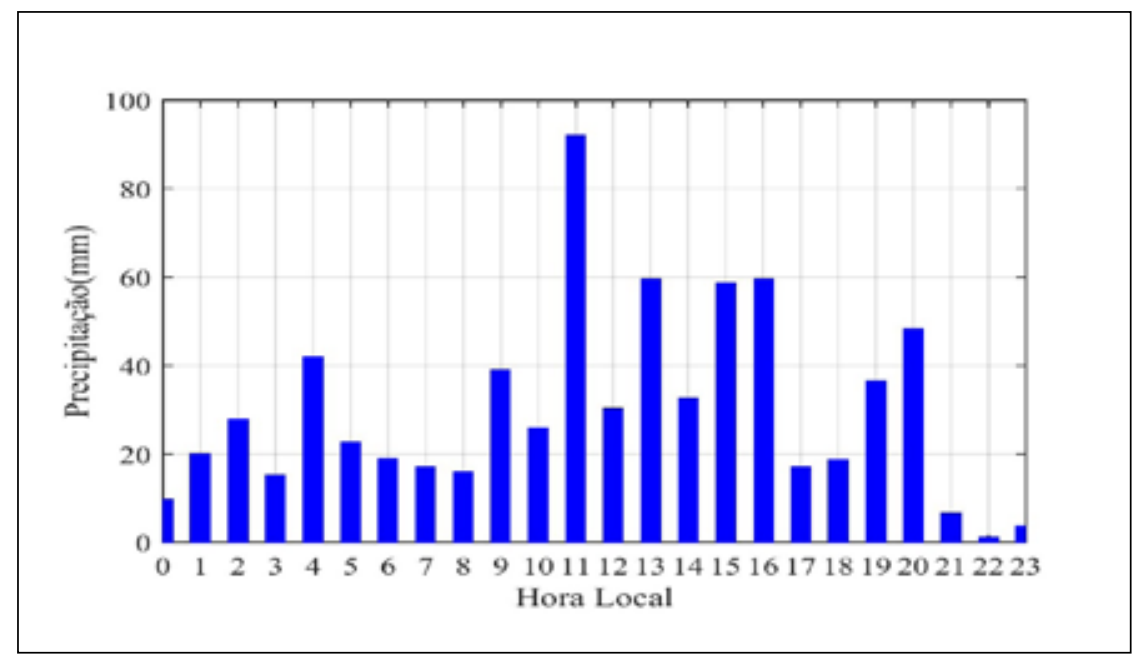

Figura 1 - Acumulado horário da precipitação (mm) durante a IOP1.

Os ciclos dos fluxos de energia a superfície são apresentados na Figura 2, onde foi possível notar que o Saldo de Radiação (Rn) apresentou valores mínimos de $-30 \mathrm{Wm}^{-2}$ durante a noite, sendo observado que, a partir das $07 \mathrm{HL}$, ocorreu uma troca de sinal de Rn para valores positivos. O máximo Rn no período foi verificado as $12 \mathrm{HL}$, com média de $420 \mathrm{Wm}^{-2} \mathrm{Porém}_{\text {, }}$ destaca-se que as entre 10 e $11 \mathrm{HL}$, verifica-se uma redução na magnitude do Rn, que pode estar associada a quantidade de precipitação ocorrida durante estes horários, e uma maior nebulosidade, em torno de 43\% na IOP1 (MACEDO e FISCH, 2017), o que acarretou em uma menor incidência de radiação de onda curta.

Ao observar o fluxo de calor latente (LE), foi verificado que mais de $85 \%$ da energia disponível é transferida para este o fluxo. Este tem seu ciclo semelhante ao do Rn, com valores praticamente nulos a noite e positivo entre 06 e 18 HL. Por se tratar de um período de observações durante a fase mais chuvosa da região, o LE demonstra máximo de $370 \mathrm{~W}^{-2}$, e assim como verificado no Rn, essa maior intensidade perdura das 12 às $13 \mathrm{HL}$.

O fluxo de Calor Sensível $(\mathrm{H})$ na estação ratifica que na Amazônia, em especial em sua fase chuvosa, apenas uma pequena fração do Rn é transferido para o fluxo de H. Entretando, salienta-se que é este fluxo que atua fortemente no desenvolvimento da CLP. Desta forma, notou-se que o $\mathrm{H}$, apresentou no período da noite valores negativos $\left(-13 \mathrm{Wm}^{-2}\right)$ e constante, começando a demonstrar resultados positivos após as $06 \mathrm{HL}$, seguindo o ciclo positivo do Rn. Entretanto, diferentemente de LE, no fluxo de $\mathrm{H}$ foi evidenciada uma elevação pouco acentuada, demonstrando em alguns momentos resultados constante entre as primeiras horas da manhã. $\mathrm{O}$ máximo de $\mathrm{H}$ foi exibido às 10:30 HL, da ordem de $50 \mathrm{Wm}^{-2}$. Esta defasagem entre o máximo de $\mathrm{H}$ com o de Rn deve-se as altas taxas de precipitação ocorrida as $11 \mathrm{HL}$.

O fluxo de calor no solo $(G)$ expôs um ciclo diário com mínimas variações durante a fase da IOP1. Nesta fase o G exibiu ciclo oposto ao do $\mathrm{H}$, com valores positivos durante a madrugada e início da manhã $\left(18,5 \mathrm{Wm}^{-2}\right)$, e ao longo do período vespertino, acarretando na maior intensidade observada às $13 \mathrm{HL}$, de $-50 \mathrm{Wm}^{-2}$, sendo esta em modulo semelhante a maior intensidade de $\mathrm{H}$. 


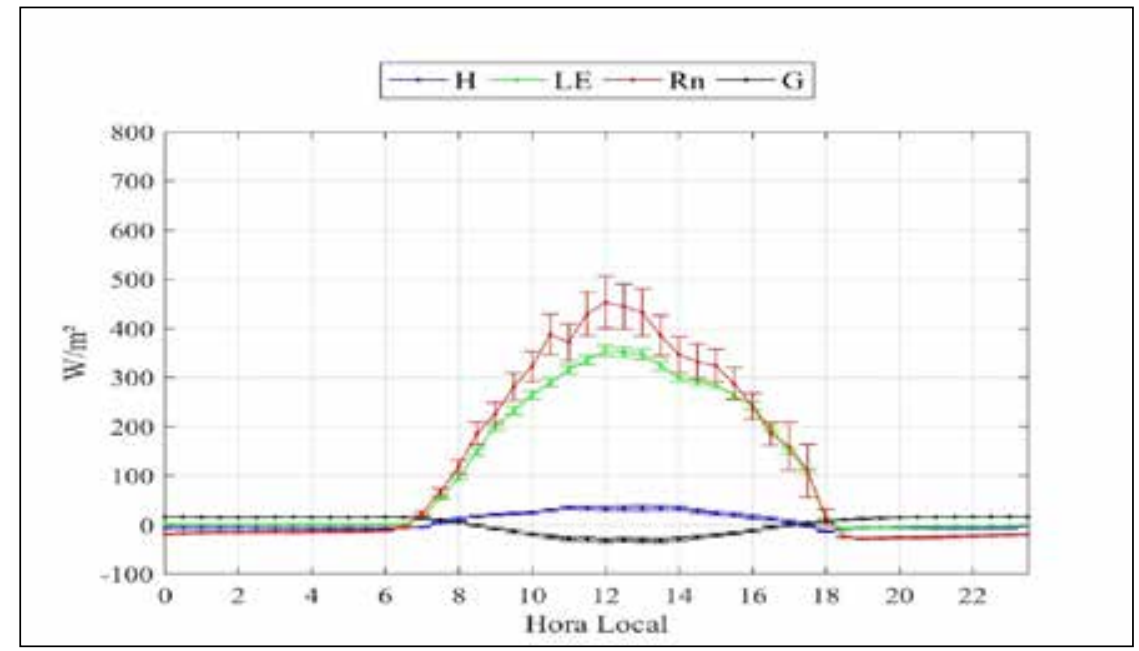

Figura 2 - Média do ciclo diário do Saldo de Radiação $(\mathrm{Rn})\left(\mathrm{W} \mathrm{m}^{-2}\right)$ (linha vermelha), Fluxo de Calor Sensível $(\mathrm{H})\left(\mathrm{W} \mathrm{m}^{-2}\right)$ (linha azul), Fluxo de Calor Latente (LE) $\left(\mathrm{W} \mathrm{m}^{-2}\right)$ (linha verde) e Fluxo de Calor no Solo $(\mathrm{G})\left(\mathrm{W} \mathrm{m}^{-2}\right)$ (linha preta) durante a fase da IOP1. Com a variabilidade dos fluxos apresentados na forma do desvio padrão.

O ciclo diário da altura da CLP na IOP1 está apresentado na Figura 3. Verificou-se que entre 00 e 06 HL a CLN encontrase formada e bem estabelecida, porém esta demonstra maiores oscilações ao longo do tempo. Nota-se, nos resultados obtidos pelos sensores remotos, foi verificado oscilações da profundidade da CLN, variando de $180 \mathrm{~m}$ (Sodar) até $450 \mathrm{~m}$ (ceilometer). Estas oscilações acarretaram em uma taxa de crescimento médio de $10,2 \mathrm{~m} \mathrm{~h}^{-1}$. A oscilação observada durante a fase da CLN pode estar relacionada com a maior ocorrência de turbulência intermitente em função do cisalhamento mecânico do vento. Enquanto que na CLN que vem a se formar no fim do dia (entre 18 e $23 \mathrm{HL}$ ), com exceção do sensor RWP, as várias determinações convergiram para um valor entre 180 e $300 \mathrm{~m}$.

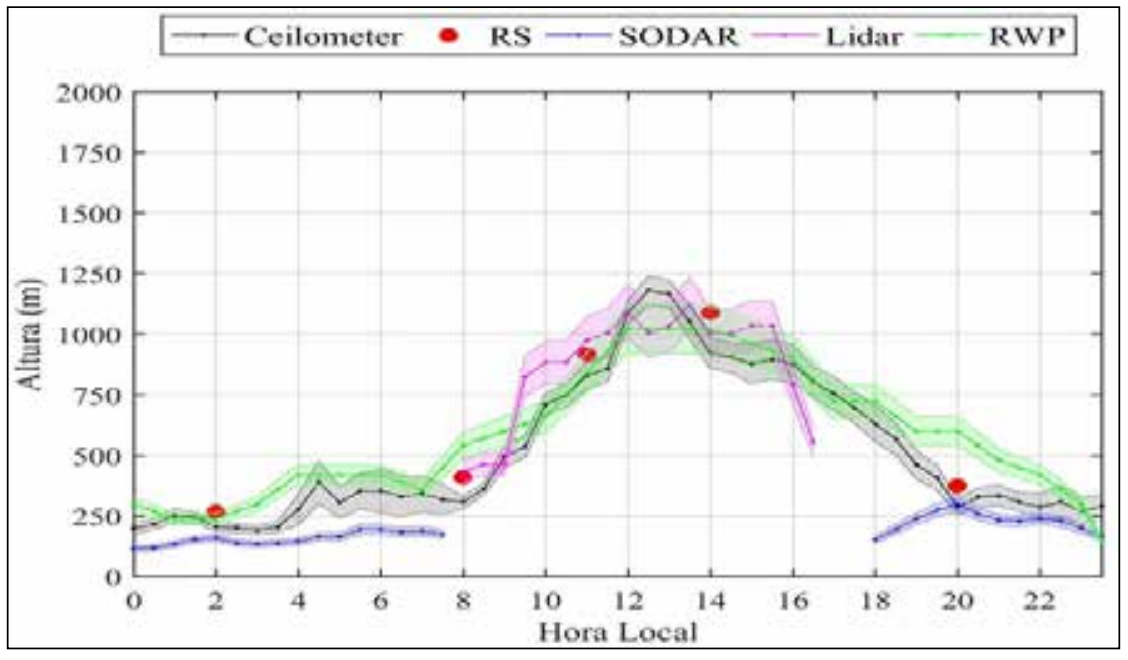

Figura 3 - Média da Altura da Camada Limite Planetária (m) durante a IOP1. As linhas verticais representam: o nascer do sol (linha vermelha) e a erosão média da CLN (linha preta).

A erosão da CLN na IOP1 ocorreu de forma lenta, tendo seu início a partir das 06 HL, quando inicia-se a incidência de radiação após o nascer do sol, entretanto, durante este período o aumento dos fluxos de superfície não foram acentuados (ver figura 2). Logo, em função da baixa elevação dos valores dos fluxos de energia nas primeiras horas do período diurno, verificou-se que até às $08 \mathrm{HL}$ não há aumento na profundidade da CLP, demonstrando que a CLN ali formada ainda não foi completamente destruída. Após às $08 \mathrm{HL}$ começa a ser registrada uma elevação da CLP, com uma taxa de crescimento médio de $22,8 \mathrm{~m} \mathrm{~h}^{-1}$. Isto ocorre devido a menor disponibilidade energética existente na IOP, o que fez com que a erosão da CLN tenha ocorrido de forma mais lenta, sendo totalmente erodida às 09:30 HL.

A fase de crescimento da CLC apresentou elevação pouco acentuada, em virtude da erosão da CLN ter ocorrida de forma lenta, demonstrando assim, a importância da erosão da CLN no que diz respeito ao início da convecção na CLP. A CLC está 
bem estabelecida às $11 \mathrm{HL}$, com $850 \mathrm{~m}$ de altura e um aumento médio de $102 \mathrm{~m} \mathrm{~h}^{-1}$, até a CLC atingir sua maior profundidade de $1.180 \mathrm{~m}$ às $13 \mathrm{HL}$. Esta profundidade está associada ao baixo valor de $\mathrm{H}$, o qual durante o ciclo diário não superara o limite máximo de $50 \mathrm{~W} \mathrm{~m}^{-2}$. Em seguida do máximo registrado, a CLC já apresenta redução em sua profundidade, com uma taxa de decaimento $-13,3 \mathrm{~m} \mathrm{~h}^{-1}$ na sua profundidade, seguindo até às $17 \mathrm{HL}$, quando os fluxos de superfície começaram a ficar negativo e, a CLN começa a se formar.

Assumindo que as medidas in situ (radiossondagem) sejam a altura da CLP e comparando com aquelas provenientes dos sensores remotos, foi possível verificar que o ceilometer demonstrou resultados coerentes com as RS, sendo que o ceilometer apresenta uma pequena subestimava $(\sim 50 \mathrm{~m})$ na fase de decaimento da convecção, estando em conformidade com resultados encontrados por Carneiro, Fisch e Kaufmann (2016). O Sodar, devido ao seu alcance vertical baixo, foi utilizado apenas para medidas na fase da CLN, em geral, subestimou $(\sim 150 \mathrm{~m})$ os valores desta. Por outro lado, o Lidar que se pode verificar a fase convectiva da CLP, apresentou medições satisfatórias, principalmente, na fase de erosão da CLN e desenvolvimento da CLC, resultados que estão de acordo com descritos por Huang et al. (2017), onde os autores afirmam que o Lidar produz valores semelhantes a profundidade da CLP durante o período de transição. Após este período este mostra uma subestimativa ( 200 $\mathrm{m})$, na fase final da CLC, onde a começa a ocorrer o decaimento da convecção. O RWP mostrou resultados em conformidade com RS durante a CLN do início do dia (entre 00 e $06 \mathrm{HL})$; nas fases de transições, o RWP mostrou uma superestimava ( 100 $\mathrm{m})$, que também foi observada na CLN que veio a se formar entre 18 a $23 \mathrm{HL}$. Este resultado do RWP está de acordo com o encontrado por Wang et al. (2016), que destacam que no período diurno as medidas baseadas no RWP têm cerca de $90 \%$ de eficácia. Entretanto, estes mesmos autores ainda afirmam que a eficácia foi reduzida para menos de $60 \%$ ao final do dia, em função da presença de uma estrutura multicamada, com a presença da camada residual (CR) e da CLN, o que vem a produzir uma falsa identificação da altura da CLP.

\section{Conclusões}

Os resultados mostraram que o ciclo diário da CLP na estação chuvosa, apresentou uma CLN com pequena (tipicamente de 300 m) oscilação em sua profundidade e uma erosão da ordem de 3 h. Na IOP chuvosa, a CLC não apresentou alta profundidade, respondendo aos baixos fluxos de $\mathrm{H}$ de superfície.

Comparando os valores de RS com os resultados obtidos pelos sensores remotos, foi verificado que o ceilometer e o RWP mostraram resultados satisfatórios da CLP. O Lidar mostrou bom resultado na fase de erosão da CLN e no desenvolvimento da CLC. Entretanto, o Sodar subestimou os valores da altura da CLN.

\section{Referências}

CARNEIRO, R. G.; FISCH, G.; KAUFMANN, T. Determinação da altura da camada limite planetária na floresta amazônica utilizando um ceilometer. Ciência e Natura, v. 38, p. 460-466, 2016.

HUANG, M.; GAO, Z.; MIAO, S.; CHEN, F.; LEMONE M. A.; HU, F. W. L. Estimate of boundary-layer depth over Beijing, china, using doppler lidar data during surf-2015. Boundary-Layer Meteorology, v. 162, p. 503-522, 2017.

MACEDO, A. S.; FISCH, G. Variabilidade temporal da radiação solar durante o experimento GOAmazon 2014/15. Revista Brasileira de Meteorologia. 2017. No prelo.

MARTIN, C.; FITZJARRALD, D.; GARSTANG, M.; OLIVEIRA, A.; GRECO, S.; BROWELL, E. Structure and growth of the mixing layer over the Amazonian rain forest. Journal of Geophysical Research, v. 93, n. D2, p. 1361-1375, 1988. ISSN 01480227.

MARTIN, S.T.; ARTAXO, P.; MACHADO, L. A. T.; MANZI, A. O.; SOUZA, R. A. F.; SCHUMACHER, C.; WANG, J.; ANDREAE, M. O.; BARBOSA, H. M. J.; FAN, J.; FISCH, G.; GOLDSTEIN, A. H.; GUENTHER, A.; JIMENEZ, J. L.; POSCHL, U.; SILVA DIAS, M. A.; SMITH, J. N.; WENDISEH, M. Introduction: Observations and Modeling of the Green Ocean Amazon (GoAmazon2014/5). Atmospheric Chemistry Physics. v. 16, p. 4785-4797, 2016.

NEVES, T. T. A. T.; FISCH, G. Camada Limite Noturna sobre área de pastagem na Amazônia. Revista Brasileira de Meteorologia, v. 26, n. 4, p. 619-628, 2011. ISSN 0102-7786. 
NEVES, T. T. A.; FISCH, G. The Daily Cycle of the Atmospheric Boundary Layer Heights over Pasture Site in Amazonia. American Journal of Environmental Engineering. v. 5, p. 39 - 44. 2015.

OUWERSLOOT, H. G.; ARELLANO, J. Vila-Guerau de; NOLSCHER, A. C.; KROL, M. C.; GANZEVELD, L. N.; BREITENBERG, C.; MAMMARELLA, I.; WILLIAMS, J.; LELIEVELD, J. Characterization of a boreal convective boundary layer and its impact on atmospheric chemistry during humppa-copec2010. Atmospheric Chemistry Physics, v. 12, p. 9335-9353, 2012.

SANTOS, R. M. N.; FISCH, G.; DOLMAN, A. J.; WATERLOO, M. Modelagem da camada limite noturna (cln) durante a época úmida na amazônia, sob diferentes condições de desenvolvimento. Revista Brasileira de Meteorologia, v. 22, p. 387-407, 2007.

SHUKLA, K. K.; PHANIKUMAR D. V.AND NEWSOM, R. K.; KUMAR, K. N.; RATNAM, M. V.; NAJA, M.; SINGH, N. Estimation of the mixing layer height over a high altitude site in central himalayan region by using doppler lidar. Journal of Atmosphericand Solar-Terrestrial Physics, v. 109, p. 48-53, 2014.

STULL, R. An introduction to boundary layer meteorology. Dordrecht: Kluwer Academic Publishers, 1988. 666 p. WANG, C.; SHI, H.; JIN, L.; CHEN, H.; WEN, H. Measuring boundary-layer height under clear and cloudy conditions using three instruments. Particuology.v. 28, p. 15 -21. 2016.

\section{Rayonil Gomes Carneiro}

Instituto Nacional de Pesquisas Espaciais , Brasil E-mail: rayonilcarneiro@gmail.com

Alice Henkes

Instituto Nacional de Pesquisas Espaciais , Brasil E-mail: alicehenkes@gmail.com

Gilberto Fisch

Instituto de Aeronáutica e Espaço, Brasil E-mail: fisch.gilberto@gmail.com

\section{Camilla Kassar Borges}

Unidade Acadêmica de Ciências Atmosféricas - Universidade Federal de Campina Grande , Brasil E-mail: camillakassar@gmail.com 